Military Technical College Kobry El-Kobbah, Cairo, Egypt

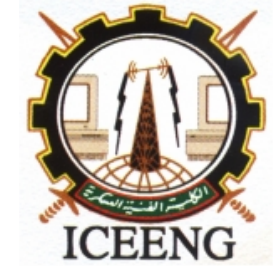

$7^{\text {th }}$ International Conference on Electrical Engineering

ICEENG 2010

\title{
Tracking and Speed Estimation of Moving Objects by Image Tracking
} Systems

By

Amr Awwad EL-Kalubi*

ZHOU Rui**

Hosam H.Abd Elhamed ***

\section{$\underline{\text { Abstract: }}$}

This paper presents an application of computer vision methods to traffic flow monitoring and road traffic analysis. The application utilizes image-processing and pattern recognition methods designed and modified to the needs and constrains of road traffic analysis. These methods combined together gives functional capabilities of the system to monitor the road, to initiate automated vehicle tracking, to measure the speed including the main steps for tracking speeds for moving objects. And design a new proposed algorithm for estimating traffic speed using a sequence of images from an un-calibrated camera using MATLAB SIMULINK.

\section{Keywords:}

Estimation, Computer Vision Systems, Image Ttracking Systems

* Egyptian Air Force

** Dept. of Automatic Control, Beihang University, Beijing, China

*** Egyptian Air Force 


\section{Introduction:}

All The development of computer vision systems has been an active research area in civilian and military applications for many years. One of the most important applications in computer vision systems is image processing and pattern recognition systems where the ability of motion estimation detection and tracking are essential to many automation tasks such as:(1) Image Processing in Road Traffic Analysis [1]. (2) Moving object detection and tracking is of great interest to many defenses Industry [2]. (3) Obstacle detection by moving object is necessary for collision avoidance, and Path planning for autonomous navigation [3].The main task of traffic monitoring applications is to identify and track the speed of moving vehicles. Many developed algorithms are applied in and approved with video monitoring system [4]. As the density of road traffic increases it becomes ever more important to detect quickly accidents or other abnormal events, both to save lives and to reduce the disruptive effects on traffic flow. Certain events can already be detected automatically using the image sequences obtained by fixed surveillance cameras, which already line many motorways and main roads. The interesting subject here is: Tracking and speed estimation of moving objects by image tracking systems.

\section{$\underline{\text { 2. Problem Description }}$}

Tracking of vehicles in images represents an important step towards achieving automated roadway monitoring capabilities. It can also be used for monitoring activities in parking lots. The challenge lies in being able to reliably and quickly detect multiple small objects of interest against a cluttered background. The problem of road monitoring is presented as a sequence of independent processing steps intended to solve tasks logically connected to each other. [2], [5], [6].These steps are (1)Video stream input to computer (personal computer or specialized one).(2)Its conversion to a sequence of single frames, lanes masking, background removal, noise and blobs filtering,(3) object contours extraction, linking and labeling, contour parameters estimation.(4)Moving vehicle tracking. (5)Velocity calculation.

Such a sequence of steps is determined by the order of logical stages. Initial data have to be given in the form of video sequence, and then processed to locate observed vehicle in each frame. The imaging procedures like segmentation, filtering, and edge detection ones are arranged and utilized, that allocate vehicle contour within frame observation zone. The next stage is to find and mark a conditional center of vehicle presented by its contour area, in order to calculate speed of an object, and to track it within frames. The third stage is to label contours, which help us to mark and calculate a number of moving objects in the observation zone, and thus estimate speeds. 


\section{Image Processing}

The tracking and speed estimation of moving vehicles, as would be used on a major highway to report traffic flow Information. This can be done in short by subtraction of the background from an input frames and creates a mask based on some error threshold. Then analyze this mask to track moving vehicles along the road. By reducing the twodimensional motion problem to the one-dimensional problem of tracking a vehicle speed can be estimated by the distance change over the time. [7], In order to make this there is a need to define a region of interest in input frames which known vehicle detection. The methods for tracking moving objects are:

- Blob Tracking.

In this approach, a background model is generated for the scene. For each input image frame, the absolute difference between the input image and the background image is processed to extract foreground blobs corresponding to the vehicles on the road[8]

- Active Contour Tracking.

A closely related approach to blob tracking is based on tracking active contours (also known as snakes) representing the boundary of an object. Vehicle tracking using active contour models has been reported by [9].

- 3D-Model Based Tracking.

Tracking vehicles using three-dimensional models has been studied by several research groups ([9], [10], and [11].Some of these approaches assume an aerial view of the scene which virtually eliminates all occlusions.

- Markov Random Field Tracking.

An algorithm for segmenting and tracking vehicles in low angle frontal sequences has been proposed by [12].In their work, the image is divided into pixel blocks, and a spatiotemporal Markov random field (ST-MRF) is used to update an object map using the current and previous image.

- Feature Tracking.

In this approach, instead of tracking a whole object, feature points on an object are tracked. The method is useful in situations of partial occlusions, where only a portion of an object is visible.[13].and [14].

- VISATRAM

This system for automatic traffic monitoring, where automatic traffic monitoring using $2 \mathrm{D}$ images. A TV camera is mounted above a highway to monitor the traffic through two slice windows, and a panoramic view image is formed for each lane. The system can count vehicles and estimate their speeds. [15]. 


\section{Mathematical model of the camera}

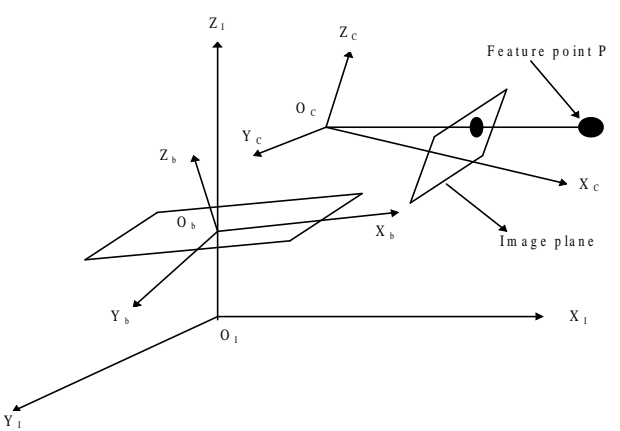

Figure 1. Types of Coordinate Systems

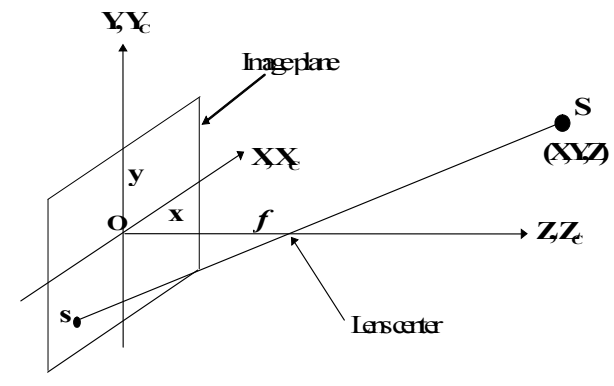

Figure 2. Image Formation Process

There are three different types of coordinate systems, Reference coordinate system, body coordinate system and camera coordinate system. As shown in figure (1). The reference coordinate system $(\mathrm{X}, \mathrm{Y}, \mathrm{Z})$ is aligned with the earth and it is fixed. (2)The body coordinate system $(\mathrm{Xb}, \mathrm{Yb}, \mathrm{Zb})$ is fixed to the centre of gravity (c.g.) of the body (platform) such as aircraft, land vehicle, robot arm. Regarding (3) the camera coordinate system (XC, YC, ZC).

- The camera coordinates $\mathrm{x}, \mathrm{y}$ can be written as:

$$
\begin{aligned}
& x=\lambda \frac{\left(X-X_{0}\right) \cos \theta+\left(Y-Y_{0}\right) \sin \theta-r_{1}}{-(X-X 0) \sin \theta \sin \alpha+(Y-Y 0) \cos \theta \sin \alpha-\left(Z-Z_{0}\right) \cos \alpha+r_{3}+\lambda} \\
& y=\lambda \frac{-\left(X-X_{0}\right) \sin \theta \cos \alpha+\left(Y-Y_{0}\right) \cos \theta \cos \phi+\left(Z-Z_{0}\right) \sin \alpha-r_{2}}{-\left(X-X_{0}\right) \sin \theta \sin \alpha+\left(Y-Y_{0}\right) \cos \theta \sin \alpha-\left(Z-Z_{0}\right) \cos \alpha+r_{3}+\lambda}
\end{aligned}
$$

The Inverse Transformation mapping used to obtain the real world coordinates point from the $2 \mathrm{D}$ image plane point by neglecting $\mathrm{Z}$ coordinate As shown in figure (2). this can be done by the following steps. So the resulting coordinates $\mathrm{X}, \mathrm{Y}$ can be written as

$$
\begin{aligned}
& X=\left(\begin{array}{l}
\left.x \cos \theta-y \sin \theta \cos \alpha+\frac{z}{\lambda}\left[r_{1} \cos \theta-r_{2} \sin \theta \cos \alpha+\left(\lambda+r_{3}\right) \sin \theta \sin \alpha+X_{0}\right]\right) \frac{\lambda}{z+\lambda} \\
+r_{1} \cos \theta-r_{2} \sin \theta \cos \alpha+r_{3} \sin \theta \sin \alpha+X_{0}
\end{array}\right. \\
& Y=\left(\begin{array}{l}
\left.x \sin \theta-y \cos \theta \cos \alpha+\frac{z}{\lambda}\left[r_{1} \sin \theta-r_{2} \cos \theta \cos \alpha+\left(\lambda+r_{3}\right) \cos \theta \sin \alpha+Y_{0}\right]\right) \frac{\lambda}{z+\lambda} \\
+r_{1} \sin \theta-r_{2} \cos \theta \cos \alpha+r_{3} \cos \theta \sin \alpha+Y_{0}
\end{array}\right. \\
& Z=\left(y \sin \alpha+\frac{z}{\lambda}\left[r_{2} \sin \alpha+\left(\lambda+r_{3}\right) \cos \alpha+Z_{0}\right]+r_{2} \sin \alpha+r_{3} \cos \alpha+Z_{0}\right) \frac{\lambda}{z+\lambda}
\end{aligned}
$$




\section{SIMULATION RESULTS OF TRACKING AND SPEED ESTIMATION}

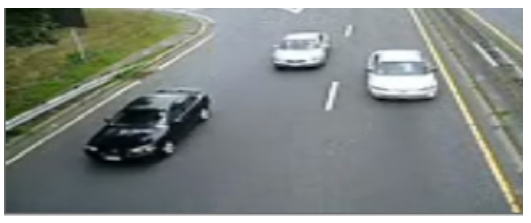

Figure 5 (a) original movie

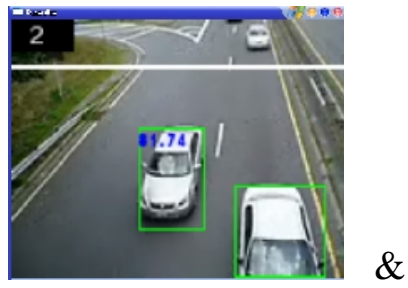

Figure 6 (a) Real traffic movie (un estimated \& estimated)

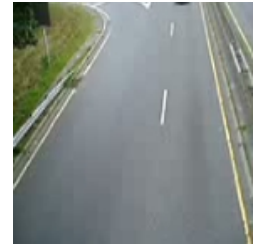

(b) background estimator

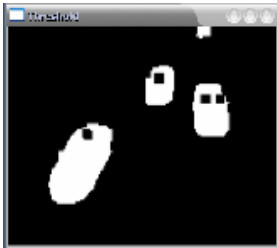

(c) segmented thresholded

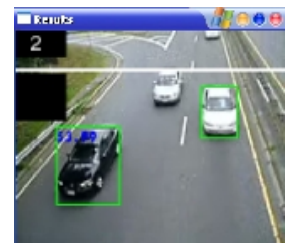

(d) counting and speed estimation
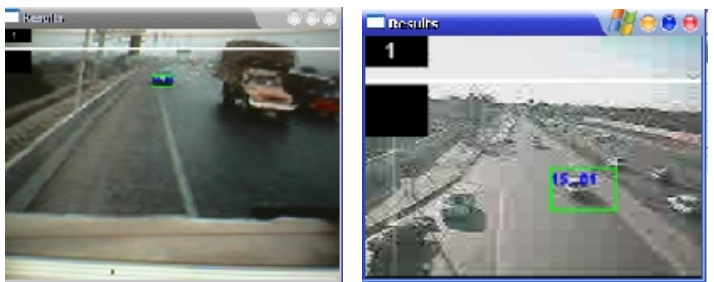

(b) Real traffic movie (taken in different roads

\begin{tabular}{|c|c|c|}
\hline \multicolumn{3}{|c|}{ Table (1) } \\
\hline Bench mark video \\
\hline Real km/h & Estimated km/h & $\begin{array}{c}\text { Error calculated } \\
(\mathrm{e})\end{array}$ \\
\hline & & $0 \%$ \\
30 & 20 & $0 \%$ \\
35 & 30 & $0 \%$ \\
40 & 35 & $0 \%$ \\
45 & 40 & $2.2 \%$ \\
50 & 46 & $2 \%$ \\
55 & 49 & $1.8 \%$ \\
60 & 54 & $3.3 \%$ \\
65 & 58 & $1.5 \%$ \\
70 & 64 & $1.4 \%$ \\
75 & 69 & $1.3 \%$ \\
80 & 74 & $2.5 \%$ \\
90 & 78 & $2.2 \%$ \\
100 & 88 & $2 \%$ \\
& 98 & \\
\hline
\end{tabular}

To test the validity of the proposed algorithm, it is applied to a benchmark video captured from a highway. The bench mark video has the true speed marked on the moving cars for reference as indicated in figure (6.a), (6.b).The result of applying the proposed algorithm to the bench mark video is summarized in table (1).From the table, it is clear that the error in the estimated speed is less than $4 \%$ for a speed varying from $30-100 \mathrm{Km} / \mathrm{hr}$ 


\section{Conclusions:}

There are two main techniques for tracking moving objects from a sequence of images. (1) Feature correspondence technique based on extracting a set of two-dimensional features in the images corresponding to three-dimensional object features in the scene, such as corners, boundaries of surfaces. (2) Optical flow technique based on distribution of apparent velocities of movement of brightness patterns in an image This paper introduces a proposed tracking system by MATLAB SIMULINK, using a sequence of images to (1)Track vehicles along the road , (2) Count number of passing vehicles and (3) Estimate Average traffic speed for each vehicle. The main outcomes of this paper is Developing the more efficient algorithm by designing a simulation model for tacking vehicles and speed estimation

\section{References:}


[1] Amar Mitichie "Computational Analysis of Visual Motion" Plenum Press, New York1994.

[2] Anil K. Jain. Fundamentals of Digital Image Processing. Prentice-Hall, 1989.

[3] J. Lipton, H. Fujiyoshi, and R. S. Patil. Moving target classification and tracking from real-time video. In IEEEWorkshop on Application of Computer Vision (WACV),pages 8-14, Princeton, NJ, 1998.

[4] Ashworth, R., D.G. Darkin, K.W. Dickinson, M.G. Hartley, C.L. Wan, and R.C.Waterfall. "Applications of Video Image Processing for Traffic Control Systems.Second International Conference on Road Traffic Control". 1985.

[5] B. Heisele and W. Ritter. Obstacle detection based on color blob flow. Proc.3th IEEE Symposium on Intelligent Vehicles, pages 282-286, 1995.

[6] Canny, J., A Computational Approach to Edge Detection. IEEE Transactions on Pattern Analysis and Machine Intelligence, 1986).

[7] C. Chui. Kalman Filtering: with "Real-time Applications, Springer Verlag”, 1991.

[8] D. A. Forsith and J. Ponce. Computer Vision-A Modern Approach. Prentice Hall, 2002.

[9] Dailey, D.J., Schoepflin T.N., 2003. A correlation technique for estimating traffic speed from cameras. Transportation Research Board Annual Meeting, TRB no. 03-3414.

[10] Dailey, D.J., Cathey, F.W., Pumrin, S., 2000. An algorithm to estimate mean traffic speed using uncalibratedcameras. IEEE Trans. on Intelligent Transportation Systems, 1:98-107.

[11] Dickinson, K.W. and R.C. Waterfall. Video Image Processing for Monitoring Road Traffic. IEE International Conference on Road Traffic Data Collection. 5-7 December 1984, London, UK, pp. 105-109.

[12] D. Koller, K. Daniilidis, and H.-H. Nagel. Model-based object tracking in monocular image sequences of road traffic scenes. International Journal of Computer Vision, 10(3):257-281, 1993.

[13] D. S. Zhang and G. Lu. A comparative study on shape retrieval using fourier descriptors with different shape signatures. In International Conference on Intelligent Multimedia and Distance Education, pages 1-9, Fargo, ND, USA, June 1-3 2001.

[14] E. R. Davies."Machine Vision: Theory, Algorithms, Practicalities". Morgan Kaufmann (2005).

[15] Francois, Alexandre and Gerard Medioni. "Adaptive Color Background Modeling for Real-Time Segmentation of Video Streams." 1996 


\section{Nomenclatures:}

Bank or Roll angle about axis OX

$\Theta$ Inclination or Pitch angle about axis OY

$\Psi \quad$ Azimuth or Yaw angle about axis $\mathrm{OZ}$ 